\title{
The Properties of Radio Selected Galaxies in HIPASS/HIJASS and SDSS
}

\author{
Diego A. Garcia-Appadoo ${ }^{1}$, Virginia A. Kilborn ${ }^{2}$, Andrew A. West ${ }^{3}$, \\ Julianne J. Dalcanton ${ }^{3}$, Mike J. Disney ${ }^{1}$ \\ ${ }^{1}$ Astronomy Department, Cardiff University, Cardiff, CF24 3YB, UK \\ ${ }^{2}$ Centre for Astrophysics, Swinburne University, Victoria 3122, \\ Australia \\ ${ }^{3}$ Department of Astronomy, University of Washington, Seattle, \\ WA98195, USA
}

\begin{abstract}
We present a preliminary analysis of the properties of HI selected galaxies selected from the HIPASS and HIJASS surveys and that fall within the SDSS. From SDSS photometry in the early data release (EDR), we have obtained optical properties such as color, morphology, surface brightness and size. By comparing these with the HI properties derived from HIPASS/HIJASS, we are able to find relationships between the cool gas and the current generation of stars.
\end{abstract}

\section{Introduction}

Understanding the relationship between neutral hydrogen (HI) and stars in the interstellar medium of galaxies is integral to the study of galaxy evolution. Because stellar populations in galaxies form from collapsed clouds of hydrogen, we can naively claim that galaxies with few stars and large quantities of gas must be less evolved than those with little gas and many stars and that galaxies evolve by converting their gas into stars. To first order this description is valid; we know that galaxies with large stellar poulations must have once had fewer stars and more gas. However, how a galaxy follows this evolutionary path is much more complicated. Factors such as mass, metal content, gas fraction, internal motion, play an important role in determining how quickly HI gets converted into stars (e.g. van Zee et al. 1998; Kennicutt 1998).

\section{Survey Details and Preliminary Results}

This project makes use of two HI surveys: the HI Parkes All Sky Survey (HIPASS; Staveley-Smith et al. 1999) covering the southern sky with an extension in the north to Decl. $<+25^{\circ}$, and the HI Jodrell All Sky Survey (HIJASS; Lang et al. 2003) which covers the rest of the northern sky. From the HI data, we can determine the position, HI mass, and rough internal kinematics for thousands of galaxies. The optical data come from the Sloan Digital Sky Survey (SDSS; Stoughton et al. 2002). SDSS will cover one-quarter of the sky in five 


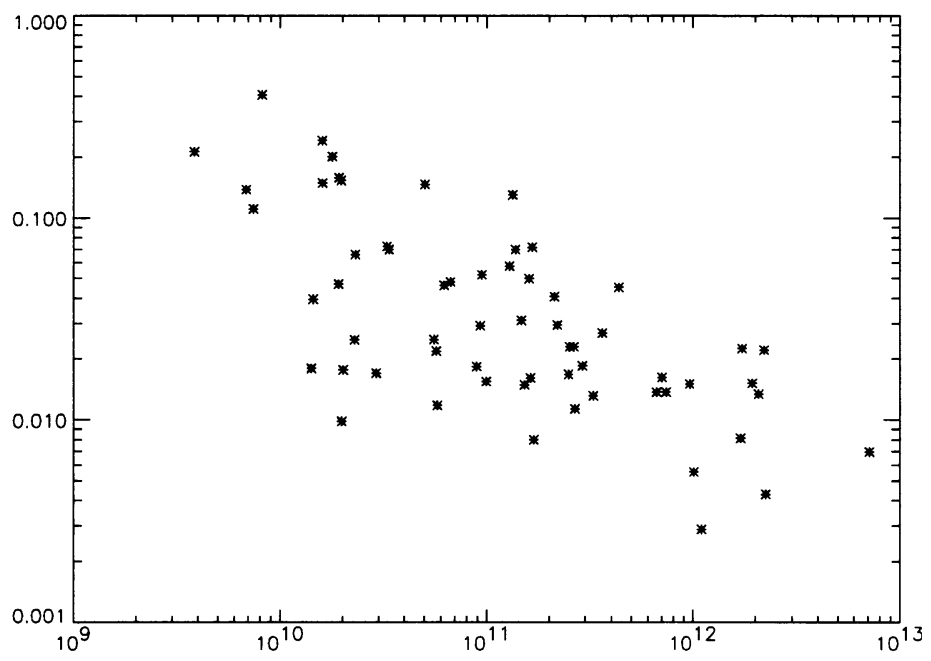

Figure 1. Ratio of HI mass to the dynamical mass versus the dynamical mass

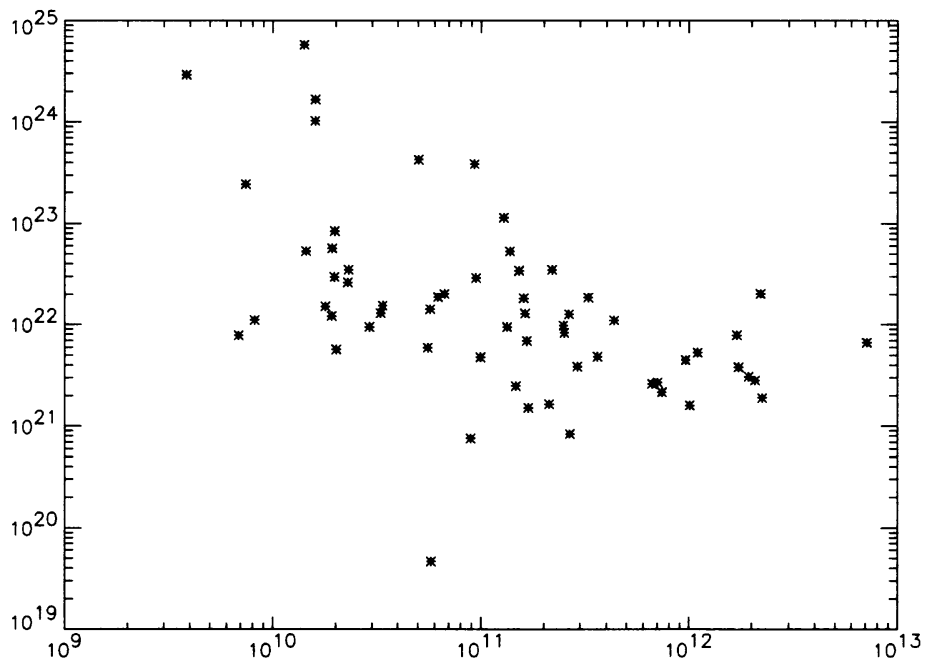

Figure 2. Ratio of HI surface density to the r-band surface brightness as a function of dynamical mass

photometric filters and will provide high quality fiber spectra.

Our study makes use of the substantial overlap between the optical and HI surveys, and allows us to investigate the ways in which neutral hydrogen correlates with stellar populations. From our preliminary study containing 80 galaxies, we can observe (Fig.1) how the HI component of low mass galaxies becomes a significant fraction of the galaxy's dynamical mass (the dynamical 
mass has been measured from the HI line width corrected for inclination). This result seems to indicate that dwarf galaxies are more Dark Matter dominated than their more massive counterparts (and less efficient at converting their gas into stars). Fig. 2 shows how lower mass systems have a proportionally higher density of gas than that of higher mass systems. These results show that gas and stellar content are important both locally and globally in low mass, dark matter dominated galaxies.

Acknowledgments. Funding for the Sloan Digital Sky Survey (SDSS) has been provided by the Alfred P. Sloan Foundation, the Participating Institutions, the National Aeronautics and Space Administration, the National Science Foundation, the U.S. Department of Energy, the Japanese Monbukagakusho, and the Max Planck Society. The SDSS Web site is http://www.sdss.org/. The SDSS is managed by the Astrophysical Research Consortium (ARC) for the Participating Institutions. The Parkes Telescope is part of the Australia Telescope which is funded by the Commonwealth of Australia for operation as a National Facility managed by CSIRO.

\section{References}

Banks G.D. et al., 1999, ApJ, 524, 612

Kennicutt, R.C.,1998, ARA\&A, 36, 189

Lang, R. H., Boyce, P. J., Kilborn, V. A. et al 2003, MNRAS, 342, 738

Stoughton et al., 2002, AJ, 123,485S

van Zee, L. et al., 1998, AJ, 116, 2805 